\title{
Nonlinear phenomena in screening of highly charged grains in plasmas
}

\author{
O.Bystrenko, A.Zagorodny \\ Bogolyubov Institute for Theoretical Physics, UA-252143 Kyiv, Ukraine
}

Received May 11, 1998

Nonlinear effects in screening a high- $Z$ grain immersed in a plasma background were investigated by means of microscopic Monte-Carlo simulations and within the Poisson-Boltzmann theory. A distinct difference from the linear Debye-Hückel approximation was detected in the case of a strong plasma-grain coupling. It effected in the accumulation of the induced plasma charge on the grain surface, accompanied by a sharp enhancement of screening. The critical value of the parameter associated with the beginning of that plasma 'condensation' was determined.

Key words: nonlinear screening, colloidal plasma, Coulomb lattice, phase transition, high-Z impurity

PACS: 52.25.Vy, 61.20.Ja, 52.25.Pp, 52.25.-b

\section{Introduction}

Colloidal plasmas, such as dusty plasmas and charged colloidal suspensions, in the recent years have attracted considerable attention as physical systems where interesting space ordering phenomena may be observed [1-3]. Typically, colloidal plasmas consist of a large number of highly charged $\left(Z \simeq 10^{3} \div 10^{6}\right)$ colloidal particles (grains) immersed in a weakly coupled plasma background. Due to a strong Coulomb repulsion between grains the colloidal component may exhibit a correlated behaviour and even form a crystalline (Coulomb) lattice. From the theoretical point of view, the simplest and fundamental questions concerning such systems are those of the effective screened field around a single high- $Z$ grain and of the effective interactions between grains. To make estimates of physical properties and even to perform Monte-Carlo (MC) or molecular dynamics computer simulations [4,5], one frequently uses as effective interactions the Yukawa-like potentials resulting from the linear Debye-Hückel (DH) screening theory. However, its validity in the case of a high- $Z$ impurity in a plasma background is a priori unclear, which can be shown as follows. The DH screening theory can be obtained as a linear approximation within the Poisson-Boltzmann (PB) approach based on 
the equation

$$
\Delta \varphi(r)-=-4 \pi Z e \delta(r)-4 \pi e n\left\{\exp \left[-\frac{e \varphi(r)}{\mathrm{k} T}\right]-\exp \left[\frac{e \varphi(r)}{\mathrm{k} T}\right]\right\}
$$

describing plasma as a two-component gas with the Boltzmann distribution. Here $\varphi$ is a screened self-consistent potential; $e$ - the charge of a positively charged plasma particle; $n$ - plasma concentration at infinity; $k$ - Boltzmann constant; $T$ - temperature.

For a spherical particle (grain) of the radius $a$, the assumption

$$
\frac{e \varphi}{\mathrm{k} T} \ll 1
$$

yields, after linearization with respect to $\varphi$, the well-known solution in the form:

$$
\varphi(r)=\frac{Z^{*} e}{r} \exp \left(-\frac{r}{r_{\mathrm{D}}}\right)
$$

with the effective charge

$$
Z^{*}=Z \frac{\exp \left(\frac{a}{r_{\mathrm{D}}}\right)}{1+\frac{a}{r_{\mathrm{D}}}},
$$

where $r_{\mathrm{D}}$ denotes the Debye screening length.

However, at short distances condition (2) is for sure violated, which makes the transition $a \rightarrow 0$ with the DH limit

$$
\varphi_{\mathrm{D}}(r)=\frac{Z e}{r} \exp \left(-\frac{r}{r_{\mathrm{D}}}\right)
$$

incorrect. This point is scarcely mentioned in literature, and relationship (5) is commonly regarded as the solution of the above problem of screening for a point charge. However, in the case of a small size grain nonlinear effects in screening might be important and a direct application of equations $(3,4)$ would fail. In that case equations $(3,4)$ would still describe the asymptotical behaviour of effective potential $\varphi$ at long distances (as condition (2) is satisfied at $r \rightarrow \infty$ ), but the value of effective charge $Z^{*}$, as well as the behaviour of function $\varphi(r)$ close to the grain surface should be found from the exact solution of equation (1). To estimate the applicability of the linear approximation, it is convenient to introduce the quantity

$$
\chi=\frac{Z e^{2}}{\mathrm{k} T a}
$$

representing the ratio of the potential energy of a plasma particle to the kinetic one on the surface of the charged grain, i.e. the maximum value of expansion parameter (2) within the domain of solution. Dusty plasmas with high $Z$ impurities and charged colloidal suspensions give examples of physical systems with $\chi$ of the order of $1 \div 10$, which casts doubt on the validity of the linear DH theory for the description of screening. 
In the present work we consider the problem of screening a finite-size charge $Z \gg 1$ in a plasma background (for the case of $\chi \simeq 1 \div 50$ ) in two ways. The first one is a correct account of nonlinear effects within the PB approach by means of a numerical solution of the associated equations. The other is the method of MC computer simulations providing a microscopic description of screening. As it follows from our computations, the both approaches indicate, at large $\chi(\chi \geqslant 5)$, and in a distinct contrast to the linear DH theory, the existence of an interesting effect associated with the accumulation of plasma charge (particles) on the surface of the grain, which sharply affects the characteristic of screening. Thus, our present aim is to show it and to compare the results obtained within the both approaches.

It should be noted that an account of nonlinear effects in the screening of high $Z$ grains within the PB theory in the context of charged colloidal suspensions was given in [6], and the results of that work correlate qualitatively with ours. Nevertheless, we performed detailed computations and presented here the results on a nonlinear PB approach along with those of MC simulations for the following reasons:

- to get a more detailed description of charge distribution and to show its critical behaviour in the vicinity of the grain surface;

- to determine the critical value of parameter $\chi$ at which sharp changes in the screening characteristic emerge;

- to make a comparison with the MC results.

The last mentioned item is of special importance. The point is that the $\mathrm{PB}$ theory is based on the concept of an effective mean field, which implies that plasmaplasma correlations must be negligibly small. A sharp concentration of plasma particles on the grain surface which accompanies the above mentioned critical effect obviously violates this requirement, which casts doubt on the nonlinear PB results altogether. For this reason the microscopic MC simulations provide significant evidence in favour of that effect.

\section{Formulation of the problem and numerical computations}

To take into account nonlinear effects within the PB approach we have to solve equation (1) numerically avoiding additional assumptions like in (2). For a hard sphere of the radius $a$ and charge $Z e$, the problem can be re-formulated with allowance for the spherical symmetry, in a dimensionless form as follows:

$$
\frac{\mathrm{d}^{2} w}{\mathrm{~d} x^{2}}=-x\left[\exp \left(-\frac{\Gamma w}{x}\right)-\exp \left(\frac{\Gamma w}{x}\right)\right] .
$$

Here $\Gamma$ is the conventional plasma coupling parameter

$$
\Gamma=\frac{e^{2}}{\mathrm{k} T d}
$$


$d=(4 \pi n)^{-1 / 3}$ is the average distance between plasma particles. Function $w$ entering equation (6) and dimensionless distance $x$ are defined via the relationships $w=r \varphi / e, x=r / d$.

The used boundary conditions were the following:

$w\left(x_{1}\right)-x_{1} w^{\prime}\left(x_{1}\right)=Z, x_{1}=a / d$;

$w\left(x_{2}\right)=0, x_{2} \rightarrow \infty$.

They define the electric field on the surface of the grain and the potential at infinity.

To find numerically the solution of equation (6), which satisfies the boundary conditions, the following shooting techniques combined with the second-order Runge-Cutta numerical algorithm were used. It can be shown that the unknown initial value $w_{1}=w\left(x_{1}\right)$ associated with the desired solution lies somewhere within the interval $(0.2 Z)$ and the solutions $w(x)$ associated with the initial values $w=0$ and $w=2 Z$ behave at $x \rightarrow \infty$, respectively, as $w \rightarrow-\infty$ and $w \rightarrow+\infty$. Therefore, the sought-for initial value $w_{1}$ and the associated solution can be found by dividing successively the interval $(0.2 Z)$ to fix the next initial value of $w$ in the centre of that interval, evaluating the corresponding solution $w(x)$ by using the Runge-Cutta algorithm, and checking the sign and the value $w_{2}$ of the constructed solution in point $x_{2}$ to determine with the desired accuracy the next sub-interval to be divided or to stop iterations if the value $w_{2}$ is zero. Point $x_{2}$ was chosen in actual calculations at sufficiently long distances, $x_{2}=3 \div 15 r_{\mathrm{D}} / d$ instead of infinity. As follows from our computations, the inaccuracy introduced by such a replacement is localized within the region of the order of $r_{\mathrm{D}}$ near the boundary point $x_{2}$. The solution in the vicinity of the grain converges therewith as the point $x_{2}$ goes to infinity, which means that the influence of the boundary condition can be made there negligibly small. The number of points dividing the domain of the solution $\left(x_{1} \leqslant x \leqslant x_{2}\right)$ was set equal to $N=10^{4}, 10^{5}, 10^{6}$.

Parameters $N$ and $x_{2}$ were chosen large enough to eliminate any dependence of the solution obtained on their values within the region $x_{1}<x<2 r_{\mathrm{D}} / d$ with the accuracy of $10^{-5}$. The computer code was tested to reproduce the exact analytical solution $(3,4)$ for the corresponding linearized boundary value problem under the same conditions. In numerical computations there were investigated:

1) effective (screened) potential $\varphi(r)$ and the ratio $\varphi(r) / \varphi_{\mathrm{D}}(r)$, - in order to find out the influence of nonlinear effects and to check the asymptotical behaviour of $\varphi(r)$ and to find the effective charge $Z^{*}$ from the relation $Z^{*}=\varphi / \varphi_{\mathrm{D}}$ at $r \gg r_{\mathrm{D}}$

2) charge distribution function $Q(r)$ defined as a total relative charge residing within a sphere of the radius $r$;

3) positive $\rho_{+}(r)$ and negative $\rho_{-}(r)$ charge densities.

Let us say a few words about the choice of parameters. The PB theory is based on the notion of the effective mean field which loses its meaning for strongly coupled $(\Gamma \geqslant 1)$ plasmas, as the plasma correlations become significant. Typical 
of colloidal plasmas are the values $Z \gg 1, \Gamma \ll 1$. Below we mainly present the results for $Z=25, \Gamma=0.1, \chi=2,10,20,40$. This choice was made, specifically, for the sake of comparison with the results of $\mathrm{MC}$ simulations, i.e. to reduce the number of the particles (very high $Z$ and very small $\Gamma$ require larger numbers of the particles).

The MC simulations of screening were performed in the model in which the two-component plasma background was represented by a sufficiently large number $(N=375)$ of charged hard spheres confined in a spherical volume with the grain of $Z=25$ fixed in the centre; the total charge of the system was equal to zero. The charge of a plasma particle and plasma temperature corresponded to the coupling constant $\Gamma=0.1$ providing the Debye screening length $r_{\mathrm{D}}=0.28$ of the radius of the spherical volume. The volume fraction of the plasma component was $v_{p}=5 \cdot 10^{-6}$, too small to reduce the plasma hard core correlations, but sufficiently large to prevent the collapsing (sticking together) of plasma particles. The radius of the central charged grain was set equal to $a / r_{\mathrm{D}}=0.61,0.11,0.047,0.017$, which was defined by the following values $\chi=2,10,20,40$.

Determination of the Debye length (as well as parameter $\Gamma$ ) in the given model represents certain difficulties, since the magnitude of plasma concentration at infinity is required. Instead of the latter, we used its average (over the spherical volume) value in the present calculations and evaluated the $\mathrm{MC}$-average magnitude of concentration at the surface of the sphere. Both treatments yielded close results, the discrepancy in $r_{D}$ did not exceed several percent.

Simulations were carried out for the NVT-ensemble using the conventional Metropolis algorithm. The amplitude of the random displacement was equal to the average distance between the particles; the walls of the sphere were considered impermeable for plasma particles. The relaxation in such a system turned out to be rather slow, that is why the first 3500000 configurations in each computer run were discarded. Every 375th of the next 3750000 configurations (to avoid statistical correlations between the configurations) was included into the ensemble, so that the total averaging was performed over 10000 statistically independent configurations.

The goal of the MC simulations was to obtain the charge distribution function $Q(r)$, defined above. Let us point out some essential differences between the formulated above models:

- the PB theory is based on the notion of the mean field, i.e. plasma correlations are totally neglected. On the contrary, MC simulations exactly take into account the microscopic structure of plasma components and plasma correlations;

- there is a distinct difference in formulation of the boundary conditions at the point $x_{2}$ in the $\mathrm{PB}$ approach and, respectively, on the surface of the sphere in $\mathrm{MC}$ simulations. In the first case the total charge density equals zero, in the second one - it is defined by a thermodynamical equilibrium and may differ from zero. As follows from our results, this circumstance is essential only in the vicinity of that boundary. 


\section{Results and conclusions}

The results of our computations are presented in figures 1-6. We formulate first the conclusions for the nonlinear PB approach and then make a comparison with the results of MC simulations.

1. As follows from our computations based on the PB approach, in all the cases the asymptotical behaviour of the screened potential at long distances has a Yukawa-like form given by equation (3), as the ratio $\varphi / \varphi_{D}$ assumes a constant at large $r$ (figure 1).

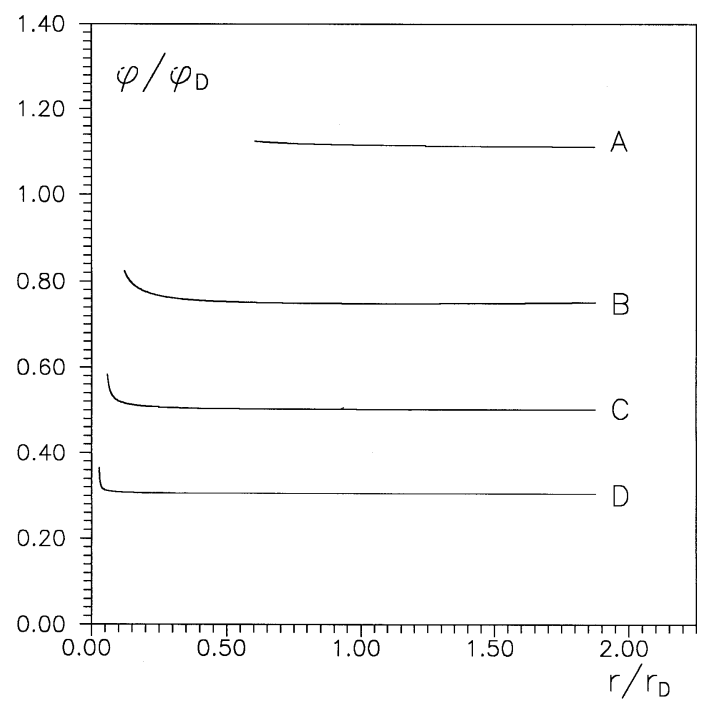

Figure 1. Ratio $\varphi / \varphi_{\mathrm{D}}$ of effective potentials $\left(\varphi\right.$ - the nonlinear $\mathrm{PB}$ approach, $\varphi_{\mathrm{D}}-$ DH approximation, equation (5)) vs. distance for $\chi=2(A), 10(B), 20(C), 40(D)$. Constant values of the ratios show the Yukawa-like asymptotical behaviour of screened potentials.

2. The value of that constant defining the effective charge $Z^{*}$ coincides with the one given by equation (4) only for small $\chi$, or, respectively, for larger radii $a$ (figures 1,2 ). Therefore, the applicability of the linearized PB theory (equations $(3,4)$ ) is limited by the condition $\chi \leqslant 1$.

3. A distinct difference between the present results and the linear DH theory was detected at large $\chi$, as nonlinear effects became more significant (figures $2-4)$. In this case the effective charge tends to diminish with growing $\chi$, so that $Z^{*} \rightarrow 0$ with $\chi \rightarrow \infty$, or, respectively, with $a / r_{\mathrm{D}} \rightarrow 0$. The screened potential in the vicinity of the grain surface loses its Yukawa-like form given by equation (3).

4. The character of charge distributions (figures 5,6) indicates that the decrease in the effective charge for large $\chi$ is accompanied by an accumulation of corresponding portions of the induced charge on the surface of a charged 
sphere; the rest of it is distributed around and produces a usual Debye-like screening at distances. The concentration of the negative plasma component on the surface may exceed its value by many orders at infinity. The point of the beginning of "condensation" in all the cases (i.e. for all sets of parameters $Z$ and $\Gamma$, etc. in our computations) was estimated as $\chi=4-6$.

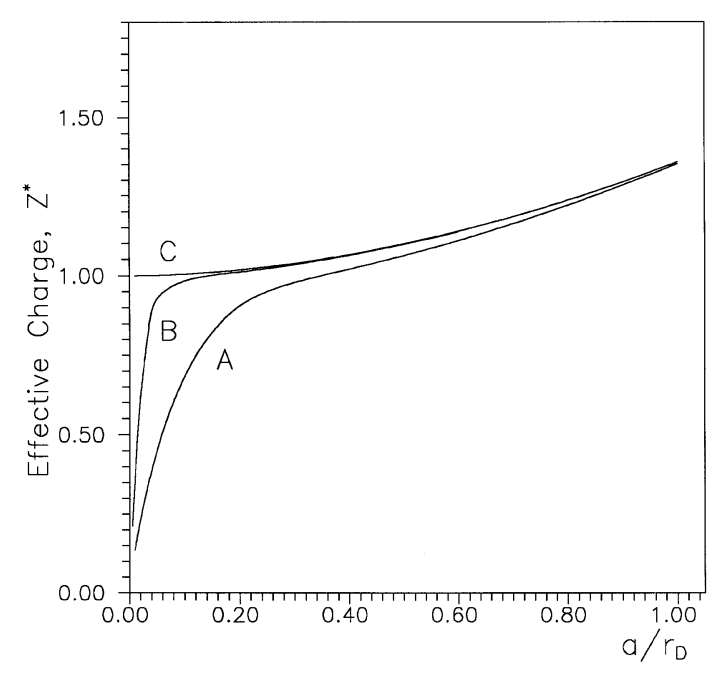

Figure 2. Effective charge $Z^{*}$ vs. diameter of the grain, defined as $Z^{*}=\varphi / \varphi_{\mathrm{D}}$ at $r \gg a . Z=25 ; \Gamma=0.1(A) ; 0.05(B)$. The line $(\mathrm{C})$ corresponds to the linear $\mathrm{DH}$ approximation (equation (4)). Account of nonlinearity gives rise to a sharp decrease in effective charge at small radii $a$.

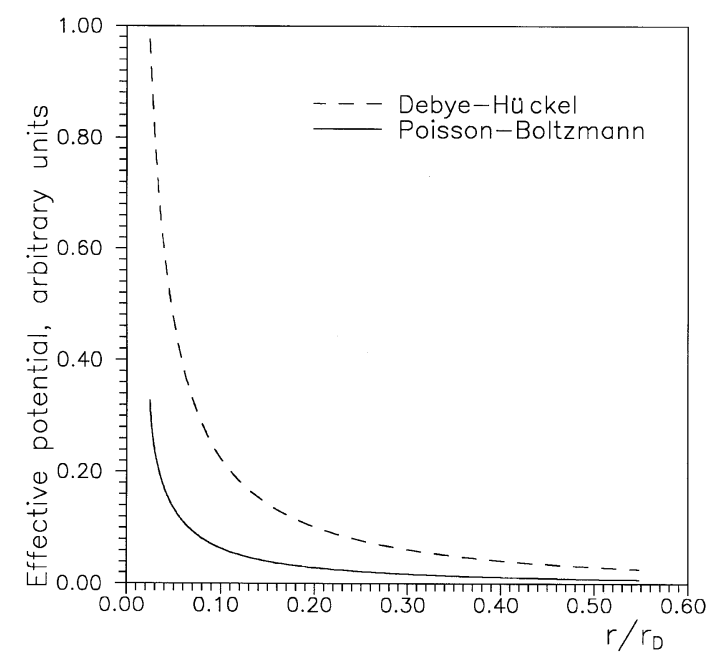

Figure 3. Effective potentials vs. distance. Solid line - the nonlinear PB approach; dashed line - the linear DH theory. $Z=25, \Gamma=0.1, \chi=45$. Nonlinearity effects in a sharp enhancement of screening.
The microscopic MC results on charge distributions in screening correlate well with those of the PB theory with allowance for nonlinearity (figure 5). Visible discrepancies between the nonlinear $\mathrm{PB}$ and $\mathrm{MC}$ results for $\chi=2 \div 20$ are still within the range of statistical errors of MC simulations which, in spite of very long chains generated, turned out to be rather considerable; the reason for that were longliving large-scale fluctuations in the given system. Marked discrepancies in the $\mathrm{MC}$ and $\mathrm{PB}$ results at larger $\chi$ $(\chi=40)$ surely do exceed statistical (MC) errors; they are apparently the consequence of the increasing effect of short-range plasma-plasma correlations in the thin layer near the surface of the grain.

The good agreement of the MC results on charge distributions with those based on the nonlinear PB approach supports the above formulated conclusions, in any event, the effect of "plasma condensation" on the grain surface at a strong plasma-grain coupling.

Apparently, the effect under consideration is significant for space ordering phenomena in colloidal plasmas, such as crystallization of a colloidal component. It considerably reduces effective interactions between the grains and may disrupt the formation of the Coulomb lattice.

It is interesting to note, that the MC simulations of infinite asymmetric two-component (colloidal) plasmas given in [7] indicated at $\chi>6$ a sort 
of peculiar condensation of plasma particles on the surface of grains. In that work this phenomenon was connected with a phase transition, especially in view of an express increase in heat capacity and the appearance of shortrange plasma-plasma correlations in this region. An apparent similarity of the results suggests that we deal with the same phenomenon, which means, in its turn, that the description of a phase transition is possible within the PB theory based on the concept of a gas in a self-consistent field. This rather unexpected conclusion may be more explainable if we recall that the given value $Z$ defining the ratio of the grain charge to that of a plasma particle introduces a microscopicity into the model and may provide a possibility of critical phenomena.

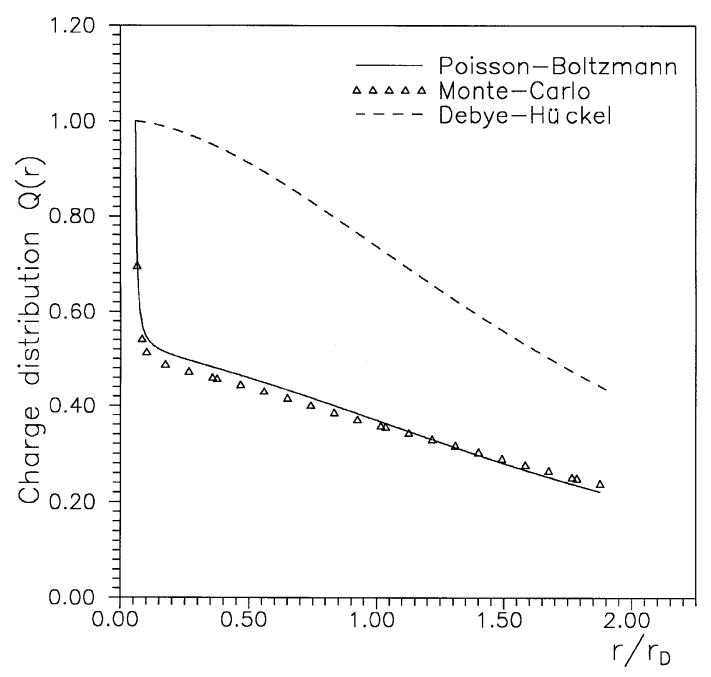

Figure 4. Comparison of charge distributions $Q(r)$ near the charged grain for $Z=25, \Gamma=0.1, \chi=20$. Dashed line the linear DH approximation; solid line - the nonlinear PB theory; symbols the results of MC simulations. A sharp step-wise change in distribution resulting from the account of nonlinearity means of an accumulation of plasma charge on the grain surface.

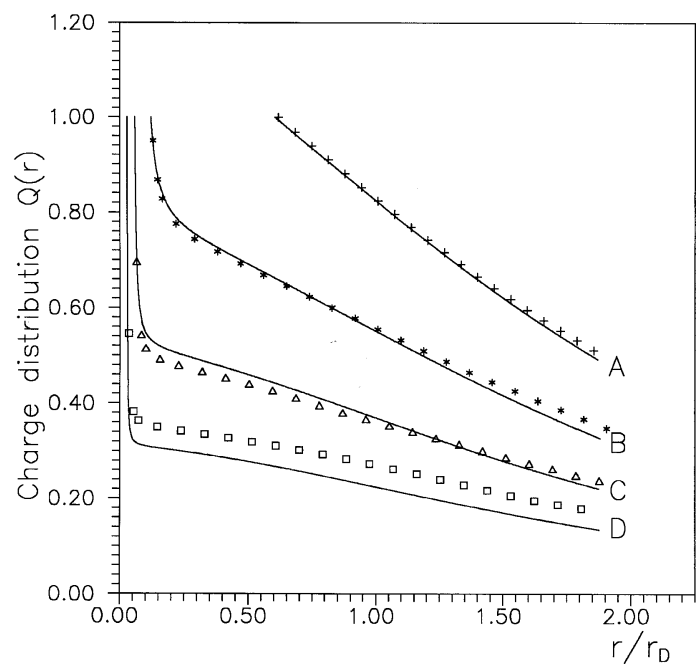

Figure 5. Comparison of charge distributions $Q(r)$ near the charged grain for $Z=25, \Gamma=0.1$. Solid lines - the nonlinear PB theory; symbols - the results of MC simulations; $\chi=2(A), 10(B), 20(C), 40(D)$. 


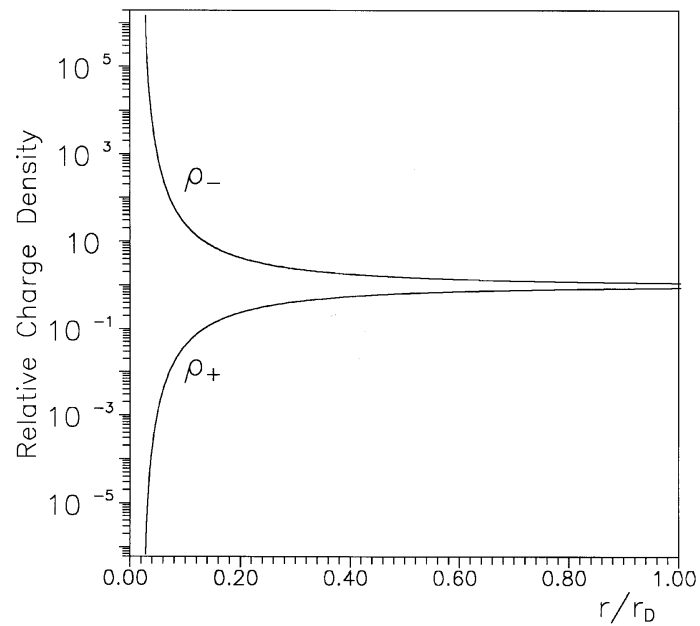

Figure 6. Positive $\rho_{+}(r)$ and negative $\rho_{-}(r)$ charge densities vs. distance. $Z=$ $25, \Gamma=0.1, \chi=40$.

\section{Acknowledgements}

We would like to thank Prof. P.Schram for valuable discussions. This work was supported in part by the State Fund of Fundamental Research of Ukraine (grant No. 2.4/319) and by the International Association INTAS (project No. 96-0617).

\section{References}

1. H.Thomas et al. Phys. Rev. Lett. 73 (1994) 652.

2. J.H.Chu, I.Lin. Physica A 205 (1994) 183.

3. P.Pieransky. Contemp. Phys. 24 (1983) 25.

4. M.O.Robbins, K.Kremer, G.S.Grest. J. Chem. Phys. 88 (1988) 286.

5. G.Dupont et al. Mol. Phys. 79 (1993) 453.

6. S.Alexander et al. J. Chem. Phys. 80 (1984) 5776.

7. O.Bystrenko, A.Zagorodny. Europhys. Conf. Abstr. 21A (1997) 1385. 


\section{Нелінійні явища в екрануванні сильнозаряджених макрочастинок у плазмі}

\section{О.Бистренко, А.Загородній}

Інститут теоретичної фізики ім. М.М.Боголюбова НАН України 252143 м. Київ, вул. Метрологічна, 146

\section{Отримано 11 травня 1998 р.}

Нелінійні ефекти в екрануванні сильнозаряджених макрочастинок, занурених у плазму, досліджено шляхом мікроскопічних моделювань методом Монте-Карло та в рамках теорії Пуасона-Больцмана. Різка відмінність від лінійного наближення Дебая-Хюкеля виявлена у випадку сильного зв'язку плазми з колоїдною частинкою. При цьому відбувається накопичення індукованого плазмового заряду на поверхні частинки, що супроводжується різким підсиленням ефекту екранування. Визначено критичне значення параметра, за якого починається плазмова конденсація.

Ключові слова: нелінійне екранування, колоїдна плазма, кулонова гратка, фазовий перехід, сильнозаряджена домішка

PACS: 52.25.Vy, 61.20.Ja, 52.25.Pp, 52.25.-b 